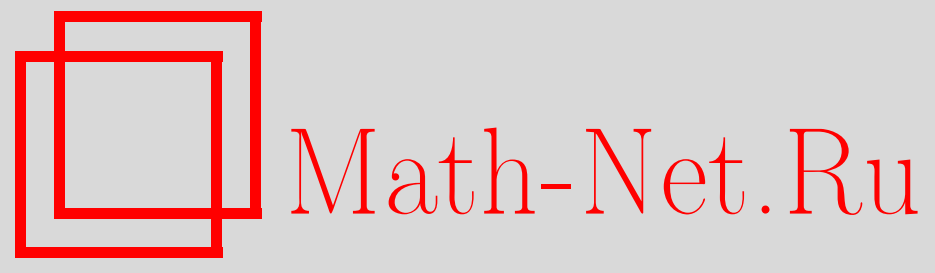

А. А. Назаров, Я. Е. Измайлова, Исследование RQ-системы с вытеснением заявок и трехфазным пофазовым дообслуживанием, Вестн. Сам. гос. техн. ун-та. Сер. Физ.-мат. науки, 2020, номер 2, 331-342

DOI: https://doi.org/10.14498/vsgtu1756

Использование Общероссийского математического портала MathNet.Ru подразумевает, что вы прочитали и согласны с пользовательским соглашением

http://www.mathnet.ru/rus/agreement

Параметры загрузки:

IP : 54.80 .73 .141

26 апреля 2023 г., 11:52:57

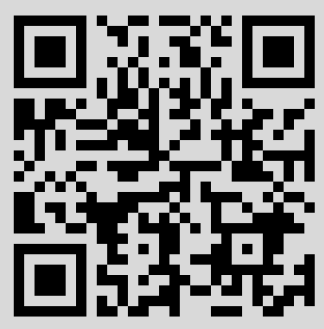


Вестн. Сам. гос. техн. ун-та. Сер. Физ.-мат. науки. 2020. Т. 24, № 2 . С. $331-342$

ISSN: 2310-7081 (online), 1991-8615 (print)

dol https://doi.org/10.14498/vsgtu1756

УДК 519.872

\title{
Исследование RQ-системы с вытеснением заявок и трехфазным пофазовым дообслуживанием
}

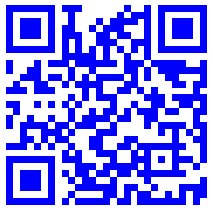

\author{
А. А. Назаров, Я. Е. Измайлова
}

Национальный исследовательский Томский государственный университет, Институт прикладной математики и компьютерных наук,

Россия, 634050, Томск, проспект Ленина, 36.

\section{Аннотация}

Рассмотрена система с повторными вызовами (RQ-система), на вход которой поступает простейший поток с заданной интенсивностью. Если в момент обращения заявки прибор занят, то происходит вытеснение заявки, стоящей на приборе. Заявка, не успевшая успешно обслужиться, переходит на орбиту, чтобы после случайной экспоненциальной задержки вновь обратиться к прибору для обслуживания. Дообслуживание заявки подразумевает, что в момент обращения с орбиты к прибору заявка встает на ту фазу обслуживания, с которой была прервана. Показано, что асимптотическая характеристическая функция числа заявок на орбите и состояний прибора сходится к трехмерному гауссовскому распределению. Для данного распределения получен вектор средних значений и матрица ковариаций. Найдено стационарное распределение вероятностей состояний прибора.

Ключевые слова: система с повторными вызовами, вытеснение заявок, трехфазное обслуживание, дообслуживание заявок, гауссовская аппроксимация, асимптотический анализ.

Получение: 18 ноября 2019 г. / Исправление: 26 мая 2020 г. / Принятие: 1 июня 2020 г. / Публикация онлайн: 3 июня 2020 г.

\section{Научная статья}

(잉 Контент публикуется на условиях лицензии Creative Commons Attribution 4.0 International (https://creativecommons.org/licenses/by/4.0/deed.ru)

\section{Образец для цитирования}

Назаров А. А., Измайлова Я. Е. Исследование RQ-системы с вытеснением заявок и трехфазным пофазовым дообслуживанием // Вестн. Сам. гос. техн. ун-та. Сер. Физ.мат. науки, 2020. Т. 24, № 2. С. 331-342. doi: 10.14498/vsgtu1756.

\section{Сведения об авторах}

Анатолий Андреевич Назаров (D) https://orcid.org/0000-0002-5097-5629

доктор технических наук, профессор; заведующий кафедрой; каф. теории вероятностей и математической статистики; e-mail: nazarov.tsu@gmail.com

Яна Евгенъевна Измайлова (1) https://orcid.org/0000-0002-9132-0127

кандидат физико-математических наук; доцент; каф. теории вероятностей и математической статистики; e-mail: evgenevna.92@mail.ru 
Введение. В последние годы активно развивается изучение систем массового обслуживания с повторами (RQ-системы). Это связано с их широким применением в различных областях: в системах телефонной коммутации, телекоммуникационных и компьютерных сетях. Ярким примером является телефонная связь. Телефонный абонент, требующий соединения и получивший сигнал «занято», будет повторять попытки до тех пор, пока не получит соединения.

Системы с повторами характеризуются тем, что заявки, прибывшие в систему и по каким-то причинам не получившие полного обслуживания, уходят в зону ожидания, называемую орбитой, и через некоторое случайное время повторяют попытку обслужиться. С целью изучения литературы по повторным очередям можно обратиться, например, к работам [1-3].

В [4] изучена система с повторными попытками, при которых прибор может предоставить дополнительную вторую фазу обслуживания. Эта модель обобщает как классическую систему с повторами, так и очередь с классической линией ожидания. K. Deka [5] изучил ненадежный сервер с двумя фазами обслуживания и повторными попытками. J.C. Ke и G. Choudhury [6] рассматривали систему с повторами, двумя фазами обслуживания, поломкой и ремонтом прибора. Также RQ-системы, в которых обслуживание осуществлялось в несколько фаз, изучены в работах $[7,8]$.

Для того чтобы предложить различное качество обслуживания для различных клиентов, мы часто устанавливаем приоритеты (то есть каким-то образом вытесняем клиентов) в системе массового обслуживания. Это явление распространено на практике. В работе [9] рассматривается система с повторной очередью, в которой приоритетом обладают только первичные заявки, то есть вытеснять в момент прихода они могут только вторичные заявки, которые обращались к прибору с орбиты. Были найдены стационарное состояние и основные показатели работоспособности системы. В [10] также рассматривают приоритетную систему, но приоритетом обладают вторичные заявки; в системе присутствует выталкивающий механизм для приоритетных заявок. Работы [11-16] также посвящены приоритетным системам.

В указанных исследованиях не учитывается тот факт, что после прерывания обслуживания не запоминается момент, с которого было прервано обслуживание, и оно начинается заново. В работе [17] показано, что при некоторых даже сколь угодно малых значениях интенсивности входящего потока стационарного режима не существует, в то время как при других параметрах этой же RQ-системы стационарный режим существует всегда при конечных значениях интенсивности входящего потока. В данной работе рассмотрим систему с повторами, где запоминается фаза, на которой прервали обслуживание, и дообслуживание начинается именно с нее.

1. Математическая модель и постановка задачи. Рассмотрим систему массового обслуживания с повторами (рис. 1). На вход поступает простейший поток заявок с интенсивностью $\lambda$. Пришедшая заявка начинает обслуживаться на первой фазе обслуживания прибора. Время обслуживания распределено экспоненциально с параметрами $\mu_{1}, \mu_{2}, \mu_{3}$ для каждой из фаз соответственно. После успешного окончания обслуживания на первой фазе заявка мгновенно переходит для обслуживания на вторую, после второйна третью и затем покидает систему. Если в момент прихода заявка обнаруживает прибор занятым, то она вытесняет заявку, стоящую на обслуживании, и занимает его. Вытесненная заявка переходит на орбиту, разделенную на три зоны. В первую зону переходят заявки с первой фазы обслуживания и воз- 


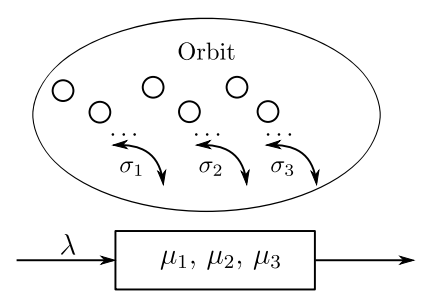

Рис. 1. Система с повторными вызовами, вытеснением заявок и трехфазным пофазовым дообслуживанием

[Figure 1. Retrial queueing system with exclusion of customers and three-phase phased by follow-up]

вращаются на дообслуживание на первую фазу, со второй зоны переходят заявки со второй фазы обслуживания и возвращаются на дообслуживание на вторую фазу, с третьей зоны - на третью. После экспоненциально распределенного времени задержки заявок на орбите с параметрами $\sigma_{1}, \sigma_{2}, \sigma_{3}$ для соответствующих зон заявки вновь встают на прибор для обслуживания. При обращении из орбиты происходит аналогичное вытеснение по тому же закону, что и для вновь прибывших в систему заявок.

Обозначим состояния прибора в момент времени $t$ следующим образом:

$$
k(t)= \begin{cases}0, & \text { если прибор пуст, } \\ 1, & \text { если прибор занят обслуживанием заявки на первой фазе, } \\ 2, & \text { если прибор занят обслуживанием заявки на второй фазе, } \\ 3, & \text { если прибор занят обслуживанием заявки на третьей фазе. }\end{cases}
$$

Пусть $P_{k}\left(i_{1}, i_{2}, i_{3}\right)=P\left\{k(t)=k, i_{1}(t)=i_{1}, i_{2}(t)=i_{2}, i_{3}(t)=i_{3}\right\}, i_{m} \geqslant 0$, $m=\overline{1,3}, k=\overline{0,3}$ - стационарное распределение вероятностей чисел заявок на орбите, где $i_{1}(t), i_{2}(t), i_{3}(t)$ - число заявок на орбите в первой, второй и третьей зонах соответственно; $R_{k}=P\{k(t)=k\}$ - стационарное распределение вероятностей состояний прибора.

Ставится задача исследования системы с повторами, вытеснением заявок и пофазовым трехфазным дообслуживанием, а именно задача нахождения стационарного распределения вероятностей состояний прибора и нахождения асимптотической характеристической функции числа заявок на орбите.

2. Система уравнений Колмогорова. Для распределения вероятностей $P_{k}\left(i_{1}, i_{2}, i_{3}\right), k=\overline{0,3}$, чисел заявок на орбите составим однородную систему с бесконечным числом линейных алгебраических уравнений Колмогоpoвa:

$$
\begin{aligned}
& -\left(\lambda+i_{1} \sigma_{1}+i_{2} \sigma_{2}+i_{3} \sigma_{3}\right) P_{0}\left(i_{1}, i_{2}, i_{3}\right)+\mu_{3} P_{3}\left(i_{1}, i_{2}, i_{3}\right)=0, \\
& -\left(\lambda+\mu_{1}+i_{2} \sigma_{2}+i_{3} \sigma_{3}\right) P_{1}\left(i_{1}, i_{2}, i_{3}\right)+\lambda P_{0}\left(i_{1}, i_{2}, i_{3}\right)+\lambda P_{1}\left(i_{1}-1, i_{2}, i_{3}\right)+ \\
& \quad+\lambda P_{2}\left(i_{1}, i_{2}-1, i_{3}\right)+\lambda P_{3}\left(i_{1}, i_{2}, i_{3}-1\right)+\left(i_{1}+1\right) \sigma_{1} P_{0}\left(i_{1}+1, i_{2}, i_{3}\right)+ \\
& \quad+\left(i_{1}+1\right) \sigma_{1} P_{2}\left(i_{1}+1, i_{2}-1, i_{3}\right)+\left(i_{1}+1\right) \sigma_{1} P_{3}\left(i_{1}+1, i_{2}, i_{3}-1\right)=0, \\
& -\left(\lambda+\mu_{2}+i_{1} \sigma_{1}+i_{3} \sigma_{3}\right) P_{2}\left(i_{1}, i_{2}, i_{3}\right)+\mu_{1} P_{1}\left(i_{1}, i_{2}, i_{3}\right)+ \\
& \quad+\left(i_{2}+1\right) \sigma_{2} P_{0}\left(i_{1}, i_{2}+1, i_{3}\right)+\left(i_{2}+1\right) \sigma_{2} P_{1}\left(i_{1}-1, i_{2}+1, i_{3}\right)+ \\
& \quad+\left(i_{2}+1\right) \sigma_{2} P_{3}\left(i_{1}, i_{2}+1, i_{3}-1\right)=0, \\
& -\left(\lambda+\mu_{3}+i_{1} \sigma_{1}+i_{2} \sigma_{2}\right) P_{3}\left(i_{1}, i_{2}, i_{3}\right)+\mu_{2} P_{2}\left(i_{1}, i_{2}, i_{3}\right)+ \\
& \quad+\left(i_{3}+1\right) \sigma_{3} P_{0}\left(i_{1}, i_{2}, i_{3}+1\right)+\left(i_{3}+1\right) \sigma_{3} P_{1}\left(i_{1}-1, i_{2}, i_{3}+1\right)+ \\
& +\left(i_{3}+1\right) \sigma_{3} P_{2}\left(i_{1}, i_{2}-1, i_{3}+1\right)=0 .
\end{aligned}
$$

Домножим правую и левую части системы (1) на величину $e^{j u_{1} i_{1}+j u_{2} i_{2}+j u_{3} i_{3}}$ и просуммируем по всем $i_{m}, m=\overline{1,3}$, где $j=\sqrt{-1}-$ мнимая единица. 
Введем функции следующего вида:

$$
H_{k}=H_{k}\left(u_{1}, u_{2}, u_{3}\right)=\sum_{i_{1}=0}^{\infty} \sum_{i_{2}=0}^{\infty} \sum_{i_{3}=0}^{\infty} e^{j u_{1} i_{1}+j u_{2} i_{2}+j u_{3} i_{3}} P_{k}\left(i_{1}, i_{2}, i_{3}\right), \quad k=\overline{0,3}
$$

Данная функция является частичной характеристической функцией.

Учитывая, что

$$
\frac{\partial H_{k}}{\partial u_{m}}=\frac{\partial H_{k}\left(u_{1}, u_{2}, u_{3}\right)}{\partial u_{m}}=\sum_{i_{1}=0}^{\infty} \sum_{i_{2}=0}^{\infty} \sum_{i_{3}=0}^{\infty} j i_{m} e^{j u_{1} i_{1}+j u_{2} i_{2}+j u_{3} i_{3}} P_{k}\left(i_{1}, i_{2}, i_{3}\right)
$$

$k=\overline{0,3}, m=\overline{1,3}$, из системы (1) следует, что для функций вида (2) уравнения Колмогорова имеют вид

$$
\begin{gathered}
j \sigma_{1} \frac{\partial H_{0}}{\partial u_{1}}+j \sigma_{2} \frac{\partial H_{0}}{\partial u_{2}}+j \sigma_{3} \frac{\partial H_{0}}{\partial u_{3}}+\mu_{3} H_{3}-\lambda H_{0}=0 \\
-\lambda H_{1}-\mu_{1} H_{1}+\lambda H_{0}+\lambda e^{j u_{1}} H_{1}+\lambda e^{j u_{2}} H_{2}+\lambda e^{j u_{3}} H_{3}+j \sigma_{3} \frac{\partial H_{1}}{\partial u_{3}}- \\
-j \sigma_{1} e^{-j u_{1}} \frac{\partial H_{0}}{\partial u_{1}}+j \sigma_{2} \frac{\partial H_{1}}{\partial u_{2}}-j \sigma_{1} e^{-j u_{1}+j u_{2}} \frac{\partial H_{2}}{\partial u_{1}}-j \sigma_{1} e^{-j u_{1}+j u_{3}} \frac{\partial H_{3}}{\partial u_{1}}=0 \\
-\lambda H_{2}-\mu_{2} H_{2}+\mu_{1} H_{1}+j \sigma_{1} \frac{\partial H_{2}}{\partial u_{1}}-j \sigma_{2} e^{-j u_{2}} \frac{\partial H_{0}}{\partial u_{2}}+j \sigma_{3} \frac{\partial H_{2}}{\partial u_{3}}- \\
-j \sigma_{2} e^{-j u_{2}+j u_{1}} \frac{\partial H_{1}}{\partial u_{2}}-j \sigma_{2} e^{-j u_{2}+j u_{3}} \frac{\partial H_{3}}{\partial u_{2}}=0 \\
-\lambda H_{3}-\mu_{3} H_{3}+\mu_{2} H_{2}+j \sigma_{1} \frac{\partial H_{3}}{\partial u_{1}}-j \sigma_{3} e^{-j u_{3}} \frac{\partial H_{0}}{\partial u_{3}}+j \sigma_{2} \frac{\partial H_{3}}{\partial u_{2}}- \\
-j \sigma_{3} e^{-j u_{3}+j u_{1}} \frac{\partial H_{1}}{\partial u_{3}}-j \sigma_{3} e^{-j u_{3}+j u_{2}} \frac{\partial H_{2}}{\partial u_{3}}=0
\end{gathered}
$$

Так как прямое решение системы (3) не представляется возможным, воспользуемся методом асимптотического анализа в предельном условии большой задержки заявок на орбите, полагая, что $\sigma_{m}=\sigma \gamma_{m}, m=1,2,3, \sigma \rightarrow 0$.

3. Асимптотический анализ. Асимптотический анализ проводится в два этапа. На первом этапе находятся стационарное распределение вероятностей состояний прибора и асимптотические средние значения числа заявок в зонах на орбите. На втором этапе находится вид предельной характеристической функции, а также параметры полученного распределения вероятностей числа заявок на орбите.

3.1. Асимптотика первого порядка. Введем следующие обозначения: $x_{1}$, $x_{2}, x_{3}$ - асимптотические средние значения числа заявок на орбите в первой, второй, третьей зонах орбиты соответственно.

Теорема 1. Пусть $i_{1}(t), i_{2}(t), i_{3}(t)$ - число заявок в зонах орбиты системы массового обслуживания с повторами, вытеснением заявок и трехфазным пофазовым дообслуживанием. Тогда выполняется предельное равенство

$$
\lim _{\sigma \rightarrow 0} M\left[\exp \left\{j u_{1} \sigma i_{1}(t)+j u_{2} \sigma i_{2}(t)+j u_{3} \sigma i_{3}(t)\right\}\right]=\exp \left\{j u_{1} x_{1}+j u_{2} x_{2}+j u_{3} x_{3}\right\},
$$


где $x_{1}, x_{2}, x_{3}$ имеют вид

$$
x_{1}=\frac{\lambda R_{1}}{\gamma_{1} R_{0}}, \quad x_{2}=\frac{\lambda R_{2}}{\gamma_{2} R_{0}}, \quad x_{3}=\frac{\lambda R_{3}}{\gamma_{3} R_{0}},
$$

a $R_{k}, k=\overline{0,3}$, определяются равенствами

$$
R_{1}=\frac{\lambda}{\mu_{1}}, \quad R_{2}=\frac{\lambda}{\mu_{2}}, \quad R_{3}=\frac{\lambda}{\mu_{3}}, \quad R_{0}=1-\frac{\lambda}{\mu_{1}}-\frac{\lambda}{\mu_{2}}-\frac{\lambda}{\mu_{3}} .
$$

Доказ а тельств о. В системе уравнений (3) выполним замены

$$
\begin{gathered}
\sigma_{m}=\sigma \gamma_{m}, \quad m=1,2,3, \quad \sigma=\epsilon, \quad u_{m}=\epsilon w_{m} \\
H_{k}=H_{k}\left(u_{1}, u_{2}, u_{3}\right)=F_{k}\left(w_{1}, w_{2}, w_{3}, \epsilon\right)=F_{k}(\epsilon), \quad k=\overline{0,3} .
\end{gathered}
$$

Тогда получаем следующую систему:

$$
\begin{gathered}
j \gamma_{1} \frac{\partial F_{0}(\epsilon)}{\partial w_{1}}+j \gamma_{2} \frac{\partial F_{0}(\epsilon)}{\partial w_{2}}+j \gamma_{3} \frac{\partial F_{0}(\epsilon)}{\partial w_{3}}+\mu_{3} F_{3}(\epsilon)-\lambda F_{0}(\epsilon)=0 \\
\lambda F_{0}(\epsilon)+\left(\lambda e^{j \epsilon w_{1}}-\lambda-\mu_{1}\right) F_{1}(\epsilon)+\lambda e^{j \epsilon w_{2}} F_{2}(\epsilon)+\lambda e^{j \epsilon w_{3}} F_{3}(\epsilon)- \\
-j \gamma_{1} e^{-j \epsilon w_{1}} \frac{\partial F_{0}(\epsilon)}{\partial w_{1}}+j \gamma_{2} \frac{\partial F_{1}(\epsilon)}{\partial w_{2}}+j \gamma_{3} \frac{\partial F_{1}(\epsilon)}{\partial w_{3}}- \\
-j \gamma_{1} e^{j \epsilon\left(w_{2}-w_{1}\right)} \frac{\partial F_{2}(\epsilon)}{\partial w_{1}}-j \gamma_{1} e^{j \epsilon\left(w_{3}-w_{1}\right)} \frac{\partial F_{3}(\epsilon)}{\partial w_{1}}=0 \\
\mu_{1} F_{1}(\epsilon)-\left(\lambda+\mu_{2}\right) F_{2}(\epsilon)-j \gamma_{2} e^{-j \epsilon w_{2}} \frac{\partial F_{0}(\epsilon)}{\partial w_{2}}-j \gamma_{2} e^{j \epsilon\left(w_{1}-w_{2}\right)} \frac{\partial F_{1}(\epsilon)}{\partial w_{2}}+ \\
+j \gamma_{1} \frac{\partial F_{2}(\epsilon)}{\partial w_{1}}+j \gamma_{3} \frac{\partial F_{2}(\epsilon)}{\partial w_{3}}-j \gamma_{2} e^{j \epsilon\left(w_{3}-w_{2}\right)} \frac{\partial F_{3}(\epsilon)}{\partial w_{2}}=0 \\
\mu_{2} F_{2}(\epsilon)-\left(\lambda+\mu_{3}\right) F_{3}(\epsilon)-j \gamma_{3} e^{-j \epsilon w_{3}} \frac{\partial F_{0}(\epsilon)}{\partial w_{3}}-j \gamma_{3} e^{j \epsilon\left(w_{1}-w_{3}\right)} \frac{\partial F_{1}(\epsilon)}{\partial w_{3}}+ \\
+j \gamma_{1} \frac{\partial F_{3}(\epsilon)}{\partial w_{1}}+j \gamma_{2} \frac{\partial F_{3}(\epsilon)}{\partial w_{2}}-j \gamma_{3} e^{j \epsilon\left(w_{2}-w_{3}\right)} \frac{\partial F_{2}(\epsilon)}{\partial w_{3}}=0 .
\end{gathered}
$$

В системе (6) выполним предельный переход при $\epsilon \rightarrow 0$ и обозначим

$$
\lim _{\epsilon \rightarrow 0} F_{k}(\epsilon)=F_{k}\left(w_{1}, w_{2}, w_{3}\right)=F_{k} .
$$

Функции $F_{k}$ будем искать в виде $F_{k}=R_{k} \Phi\left(w_{1}, w_{2}, w_{3}\right)=R_{k} \Phi$. После всех замен и предельного перехода из системы (6) получаем

$$
\begin{array}{r}
\left(-\lambda R_{0}+\mu_{3} R_{3}\right) \Phi+j R_{0}\left(\gamma_{1} \frac{\partial \Phi}{\partial w_{1}}+\gamma_{2} \frac{\partial \Phi}{\partial w_{2}}+\gamma_{3} \frac{\partial \Phi}{\partial w_{3}}\right)=0 \\
\left(\lambda\left(1-R_{1}\right)-\mu_{1} R_{1}\right) \Phi-j \gamma_{1}\left(1-R_{1}\right) \frac{\partial \Phi}{\partial w_{1}}+j \gamma_{2} R_{1} \frac{\partial \Phi}{\partial w_{2}}+j \gamma_{3} R_{1} \frac{\partial \Phi}{\partial w_{3}}=0 \\
\left(\left(-\lambda+\mu_{2}\right) R_{2}+\mu_{1} R_{1}\right) \Phi-j \gamma_{2}\left(1-R_{2}\right) \frac{\partial \Phi}{\partial w_{2}}+j \gamma_{1} R_{2} \frac{\partial \Phi}{\partial w_{1}}+j \gamma_{3} R_{2} \frac{\partial \Phi}{\partial w_{3}}=0, \\
\left(\left(-\lambda+\mu_{3}\right) R_{3}+\mu_{2} R_{2}\right) \Phi-j \gamma_{3}\left(1-R_{3}\right) \frac{\partial \Phi}{\partial w_{3}}+j \gamma_{1} R_{3} \frac{\partial \Phi}{\partial w_{1}}+j \gamma_{2} R_{3} \frac{\partial \Phi}{\partial w_{2}}=0 .
\end{array}
$$


Левые и правые части уравнений полученной системы разделим на $\Phi$ и эту функцию будем искать в виде

$$
\Phi=\exp \left(j w_{1} x_{1}+j w_{2} x_{2}+j w_{3} x_{3}\right)
$$

Тогда получаем систему уравнений

$$
\begin{array}{r}
-\left(\lambda+\gamma_{1} x_{1}+\gamma_{2} x_{2}+\gamma_{3} x_{3}\right) R_{0}+\mu_{3} R_{3}=0 \\
\left(\lambda+\gamma_{1} x_{1}\right) R_{0}-\left(\mu_{1}+\gamma_{2} x_{2}+\gamma_{3} x_{3}\right) R_{1}+\left(\lambda+\gamma_{1} x_{1}\right)\left(R_{2}+R_{3}\right)=0 \\
\gamma_{2} x_{2} R_{0}+\left(\mu_{1}+\gamma_{2} x_{2}\right) R_{1}-\left(\lambda+\mu_{2}+\gamma_{1} x_{1}+\gamma_{3} x_{3}\right) R_{2}+\gamma_{2} x_{2} R_{3}=0 \\
\gamma_{3} x_{3} R_{0}+\gamma_{3} x_{3} R_{1}+\left(\mu_{2}+\gamma_{3} x_{3}\right) R_{2}-\left(\lambda+\mu_{3}+\gamma_{1} x_{1}+\gamma_{2} x_{2}\right) R_{3}=0
\end{array}
$$

Просуммируем уравнения системы (6), выполним предельный переход при $\epsilon \rightarrow 0$ и, учитывая (7), (9), получим

$$
\begin{aligned}
& \left(-\gamma_{1} x_{1} R_{0}+\left(\lambda+\gamma_{2} x_{2}+\gamma_{3} x_{3}\right) R_{1}-\gamma_{1} x_{1}\left(R_{2}+R_{3}\right)\right) w_{1}+ \\
& \quad+\left(-\gamma_{2} x_{2} R_{0}+\left(\lambda+\gamma_{1} x_{1}+\gamma_{3} x_{3}\right) R_{2}-\gamma_{2} x_{2}\left(R_{1}+R_{3}\right)\right) w_{2}+ \\
& \quad+\left(-\gamma_{3} x_{3} R_{0}+\left(\lambda+\gamma_{1} x_{1}+\gamma_{2} x_{2}\right) R_{3}-\gamma_{3} x_{3}\left(R_{1}+R_{2}\right)\right) w_{3}=0
\end{aligned}
$$

Приравнивая коэффициенты при $w_{m}, m=\overline{1,3}$, к нулю, получим систему

$$
\begin{aligned}
& -\gamma_{1} x_{1} R_{0}+\left(\lambda+\gamma_{2} x_{2}+\gamma_{3} x_{3}\right) R_{1}-\gamma_{1} x_{1}\left(R_{2}+R_{3}\right)=0 \\
& -\gamma_{2} x_{2} R_{0}+\left(\lambda+\gamma_{1} x_{1}+\gamma_{3} x_{3}\right) R_{2}-\gamma_{2} x_{2}\left(R_{1}+R_{3}\right)=0 \\
& -\gamma_{3} x_{3} R_{0}+\left(\lambda+\gamma_{1} x_{1}+\gamma_{2} x_{2}\right) R_{3}-\gamma_{3} x_{3}\left(R_{1}+R_{2}\right)=0
\end{aligned}
$$

объединяя которую с системой (9) и используя условие нормировки $\sum_{k=0}^{3} R_{k}=1$, получаем формулы (4) и (5).

Таким образом, при малых значениях $\sigma$ среднее число заявок на орбите приближенно равно $\left(x_{1}+x_{2}+x_{3}\right) / \sigma$.

3.2. Асимптотика второго порядка. Построим гауссовскую аппроксимацию числа заявок на орбите.

Пусть

$$
\mathbf{K}=\left[\begin{array}{lll}
K_{11} & K_{12} & K_{13} \\
K_{21} & K_{22} & K_{23} \\
K_{31} & K_{32} & K_{33}
\end{array}\right]
$$

- матрица ковариаций.

С учетом того, что $K_{12}=K_{21}, K_{13}=K_{31}, K_{23}=K_{32}$, запишем вектор $\mathbf{k}$ :

$$
\mathbf{k}=\left[\begin{array}{llllll}
K_{11} & K_{12} & K_{13} & K_{22} & K_{23} & K_{33}
\end{array}\right]
$$

Tеорема 2. Пусть $i_{1}(t), i_{2}(t), i_{3}(t)$ - число заявок в зонах орбиты системы массового обслуживания с повторами, вытеснением заявок и трехфазным пофазовым дообслуживанием. Тогда выполняется предельное равенство

$$
\lim _{\sigma \rightarrow 0} M\left[\exp \left\{\sum_{\nu=1}^{3} j u_{\nu} \sqrt{\sigma}\left\{i_{\nu}(t)-\frac{x_{\nu}}{\sigma}\right\}\right\}\right]=\exp \left\{\frac{1}{2} \sum_{\nu=1}^{3} \sum_{\kappa=1}^{3} j u_{\kappa} j u_{\nu} K_{\kappa \nu}\right\},
$$


где $x_{\nu}, \nu=\overline{1,3}$, имеют вид (4), а элементы матрицы ковариаций $K_{\kappa \nu}, \nu=$ $=\overline{1,3}, \kappa=\overline{1,3}$, находятся из матричного уравнения

$$
\mathbf{k A}=\mathbf{C}
$$

в котором элементы матрицы $A_{\kappa \nu}, \nu=\overline{1,6}, \kappa=\overline{1,6}$, имеют вид

$$
\begin{aligned}
& A_{11}=-\gamma_{1}\left(R_{0} z_{0}+R_{2} z_{2}+R_{3} z_{3}\right)+\gamma_{1}\left(1-R_{1}\right)\left(1+z_{1}\right) \text {, } \\
& A_{21}=\gamma_{2} R_{1}+\gamma_{2} z_{2}\left(1-R_{2}\right)-\gamma_{2}\left(R_{0} z_{0}+R_{1} z_{1}+R_{3} z_{3}\right) \text {, } \\
& A_{31}=\gamma_{3} z_{3}\left(1-R_{3}\right)-\gamma_{3}\left(R_{0} z_{0}+R_{1} z_{1}+R_{2} z_{2}\right)-\gamma_{3} R_{1} \text {, } \\
& A_{22}=\gamma_{1} R_{2}+\gamma_{1} a_{1}\left(1-R_{1}\right)-\gamma_{1}\left(R_{0} a_{0}+R_{2} a_{2}+R_{3} a_{3}\right) \text {, } \\
& A_{42}=-\gamma_{2}\left(R_{0} a_{0}+R_{1} a_{1}+R_{3} a_{3}\right)+\gamma_{2}\left(1-R_{2}\right)\left(1+a_{2}\right) \text {, } \\
& A_{52}=\gamma_{3} a_{3}\left(1-R_{3}\right)-\gamma_{3}\left(R_{0} a_{0}+R_{1} a_{1}+R_{2} a_{2}\right)-\gamma_{3} R_{2} \text {, } \\
& A_{33}=\gamma_{1} s_{1}\left(1-R_{1}\right)-\gamma_{1}\left(R_{0} s_{0}+R_{2} s_{2}+R_{3} s_{3}\right)-\gamma_{1} R_{3} \text {, } \\
& A_{53}=\gamma_{2} R_{3}+\gamma_{2} s_{2}\left(1-R_{2}\right)-\gamma_{2}\left(R_{0} s_{0}+R_{1} s_{1}+R_{3} s_{3}\right) \text {, } \\
& A_{63}=-\gamma_{3}\left(R_{0} s_{0}+R_{1} s_{1}+R_{2} s_{2}\right)+\gamma_{3}\left(1-R_{3}\right)\left(1+s_{3}\right) \text {, } \\
& A_{14}=\gamma_{1} a_{1}\left(1-R_{1}\right)-\gamma_{1}\left(R_{0} a_{0}+R_{2} a_{2}+R_{3} a_{3}\right)-\gamma_{1} R_{2} \text {, } \\
& A_{24}=-\gamma_{1}\left(R_{0} z_{0}+R_{2} z_{2}+R_{3} z_{3}\right)+\gamma_{1}\left(1-R_{1}\right)\left(1+z_{1}\right) \\
& -\gamma_{2}\left(R_{0} a_{0}+R_{1} a_{1}+R_{3} a_{3}\right)+\gamma_{2}\left(1-R_{2}\right)\left(1+a_{2}\right), \\
& A_{34}=\gamma_{3} a_{3}\left(1-R_{3}\right)-\gamma_{3}\left(R_{0} a_{0}+R_{1} a_{1}+R_{2} a_{2}\right)-\gamma_{3} R_{2} \text {, } \\
& A_{44}=\gamma_{2} z_{2}\left(1-R_{2}\right)-\gamma_{2}\left(R_{0} z_{0}+R_{1} z_{1}+R_{3} z_{3}\right)-\gamma_{2} R_{1} \text {, } \\
& A_{54}=\gamma_{3} z_{3}\left(1-R_{3}\right)-\gamma_{3}\left(R_{0} z_{0}+R_{1} z_{1}+R_{2} z_{2}\right)-\gamma_{3} R_{1} \text {, } \\
& A_{15}=\gamma_{1} s_{1}\left(1-R_{1}\right)-\gamma_{1}\left(R_{0} s_{0}+R_{2} s_{2}+R_{3} s_{3}\right)-\gamma_{1} R_{3} \text {, } \\
& A_{25}=\gamma_{2} s_{2}\left(1-R_{2}\right)-\gamma_{2}\left(R_{0} s_{0}+R_{1} s_{1}+R_{3} s_{3}\right)-\gamma_{2} R_{3} \text {, } \\
& A_{35}=-\gamma_{1}\left(R_{0} z_{0}+R_{2} z_{2}+R_{3} z_{3}\right)+\gamma_{1}\left(1-R_{1}\right)\left(1+z_{1}\right)- \\
& -\gamma_{3}\left(R_{0} s_{0}+R_{1} s_{1}+R_{2} s_{2}\right)+\gamma_{3}\left(1-R_{3}\right)\left(1+s_{3}\right), \\
& A_{55}=\gamma_{2} z_{2}\left(1-R_{2}\right)-\gamma_{2}\left(R_{0} z_{0}+R_{1} z_{1}+R_{3} z_{3}\right)-\gamma_{2} R_{1} \text {, } \\
& A_{65}=\gamma_{3} z_{3}\left(1-R_{3}\right)-\gamma_{3}\left(R_{0} z_{0}+R_{1} z_{1}+R_{2} z_{2}\right)-\gamma_{3} R_{1} \text {, } \\
& A_{26}=\gamma_{1} s_{1}\left(1-R_{1}\right)-\gamma_{1}\left(R_{0} s_{0}+R_{2} s_{2}+R_{3} s_{3}\right)-\gamma_{1} R_{3} \text {, } \\
& A_{36}=\gamma_{1} a_{1}\left(1-R_{1}\right)-\gamma_{1}\left(R_{0} a_{0}+R_{2} a_{2}+R_{3} a_{3}\right)-\gamma_{1} R_{2} \text {, } \\
& A_{46}=\gamma_{2} s_{2}\left(1-R_{2}\right)-\gamma_{2}\left(R_{0} s_{0}+R_{1} s_{1}+R_{3} s_{3}\right)-\gamma_{2} R_{3} \text {, } \\
& A_{56}=-\gamma_{2}\left(R_{0} a_{0}+R_{1} a_{1}+R_{3} a_{3}\right)+\gamma_{2}\left(1-R_{2}\right)\left(1+a_{2}\right)- \\
& -\gamma_{3}\left(R_{0} s_{0}+R_{1} s_{1}+R_{2} s_{2}\right)+\gamma_{3}\left(1-R_{3}\right)\left(1+s_{3}\right) \text {, } \\
& A_{66}=\gamma_{3} a_{3}\left(1-R_{3}\right)-\gamma_{3}\left(R_{0} a_{0}+R_{1} a_{1}+R_{2} a_{2}\right)-\gamma_{3} R_{2} \text {, } \\
& A_{13}=0, \quad A_{12}=0, \quad A_{23}=0, \quad A_{43}=0, \quad A_{41}=0, \quad A_{51}=0, \\
& A_{61}=0, \quad A_{32}=0, \quad A_{62}=0, \quad A_{64}=0, \quad A_{45}=0, \quad A_{16}=0 \text {, }
\end{aligned}
$$

а элементы вектора $\mathbf{C}$ имеют следующий вид:

$$
\begin{aligned}
& C_{1}=\frac{\gamma_{1} x_{1}}{2}\left(1-R_{1}\right)+\left(\lambda+\gamma_{2} x_{2}+\gamma_{3} x_{3}\right) \frac{R_{1}}{2}- \\
& -\lambda R_{1} z_{1}+\gamma_{1} x_{1} z_{1}\left(1-R_{1}\right)-\gamma_{2} x_{2} z_{2} R_{1}-\gamma_{3} x_{3} z_{3} R_{1}, \\
& C_{2}=\frac{\gamma_{2} x_{2}}{2}\left(1-R_{2}\right)+\left(\lambda+\gamma_{1} x_{1}+\gamma_{3} x_{3}\right) \frac{R_{2}}{2}- \\
& -\lambda R_{2} a_{1}+\gamma_{2} x_{2} a_{2}\left(1-R_{2}\right)-\gamma_{1} x_{1} a_{1} R_{2}-\gamma_{3} x_{3} a_{3} R_{2}, \\
& C_{3}=\frac{\gamma_{3} x_{3}}{2}\left(1-R_{3}\right)+\left(\lambda+\gamma_{1} x_{1}+\gamma_{2} x_{2}\right) \frac{R_{3}}{2}- \\
& -\lambda R_{3} s_{1}+\gamma_{3} x_{3} s_{3}\left(1-R_{3}\right)-\gamma_{1} x_{1} s_{1} R_{3}-\gamma_{2} x_{2} s_{2} R_{3}, \\
& C_{4}=-\gamma_{1} x_{1} R_{2}-\gamma_{2} x_{2} R_{1}-\lambda R_{1} a_{1}+\gamma_{1} x_{1} a_{1}\left(1-R_{1}\right)-\gamma_{2} x_{2} R_{1} a_{2}- \\
& -\gamma_{3} x_{3} R_{1} a_{3}-\lambda R_{2} z_{1}-\gamma_{1} x_{1} R_{2} z_{1}+\gamma_{2} x_{2} z_{2}\left(1-R_{2}\right)-\gamma_{3} x_{3} R_{2} z_{3} \text {, }
\end{aligned}
$$




$$
\begin{aligned}
C_{5}= & -\gamma_{1} x_{1} R_{3}-\gamma_{3} x_{3} R_{1}-\lambda R_{1} s_{1}+\gamma_{1} x_{1} s_{1}\left(1-R_{1}\right)-\gamma_{2} x_{2} R_{1} s_{2}- \\
& -\gamma_{3} x_{3} R_{1} s_{3}-\lambda R_{3} z_{1}-\gamma_{1} x_{1} R_{3} z_{1}+\gamma_{3} x_{3} z_{3}\left(1-R_{3}\right)-\gamma_{2} x_{2} R_{3} z_{2} \\
C_{6}= & -\gamma_{2} x_{2} R_{3}-\gamma_{3} x_{3} R_{2}-\lambda R_{2} s_{1}+\gamma_{2} x_{2} s_{2}\left(1-R_{2}\right)-\gamma_{1} x_{1} R_{2} s_{1}- \\
& -\gamma_{3} x_{3} R_{2} s_{3}-\lambda R_{3} a_{1}-\gamma_{1} x_{1} R_{3} a_{1}+\gamma_{3} x_{3} a_{3}\left(1-R_{3}\right)-\gamma_{2} x_{2} R_{3} a_{2}
\end{aligned}
$$

Величины $z_{k}, a_{k}, s_{k}, k=\overline{0,3}$, находятся из следующих систем уравнений:

- система для нахождения значений $z_{k}$ :

$-\left(\lambda+\gamma_{1} x_{1}+\gamma_{2} x_{2}+\gamma_{3} x_{3}\right) z_{0}+\left(\lambda+\gamma_{1} x_{1}\right) z_{1}+\gamma_{2} x_{2} z_{2}+\gamma_{3} x_{3} z_{3}=-\gamma_{1} x_{1}$, $-\left(\mu_{1}+\gamma_{2} x_{2}+\gamma_{3} x_{3}\right) z_{1}+\left(\mu_{1}+\gamma_{2} x_{2}\right) z_{2}+\gamma_{3} x_{3} z_{3}=\lambda+\gamma_{2} x_{2}+\gamma_{3} x_{3}$,

$\left(\lambda+\gamma_{1} x_{1}\right) z_{1}-\left(\lambda+\mu_{2}+\gamma_{1} x_{1}+\gamma_{3} x_{3}\right) z_{2}+\left(\mu_{2}+\gamma_{3} x_{3}\right) z_{3}=-\gamma_{1} x_{1}$, $\mu_{3} z_{0}+\left(\lambda+\gamma_{1} x_{1}\right) z_{1}+\gamma_{2} x_{2} z_{2}-\left(\lambda+\mu_{3}+\gamma_{1} x_{1}+\gamma_{2} x_{2}\right) z_{3}=-\gamma_{1} x_{1}$;

- система для нахождения значений $a_{k}$ :

$-\left(\lambda+\gamma_{1} x_{1}+\gamma_{2} x_{2}+\gamma_{3} x_{3}\right) a_{0}+\left(\lambda+\gamma_{1} x_{1}\right) a_{1}+\gamma_{2} x_{2} a_{2}+\gamma_{3} x_{3} a_{3}=-\gamma_{2} x_{2}$, $-\left(\mu_{1}+\gamma_{2} x_{2}+\gamma_{3} x_{3}\right) a_{1}+\left(\mu_{1}+\gamma_{2} x_{2}\right) a_{2}+\gamma_{3} x_{3} a_{3}=-\gamma_{2} x_{2}$,

$\left(\lambda+\gamma_{1} x_{1}\right) a_{1}-\left(\lambda+\mu_{2}+\gamma_{1} x_{1}+\gamma_{3} x_{3}\right) a_{2}+\left(\mu_{2}+\gamma_{3} x_{3}\right) a_{3}=\lambda+\gamma_{1} x_{1}+\gamma_{3} x_{3}$, $\mu_{3} a_{0}+\left(\lambda+\gamma_{1} x_{1}\right) a_{1}+\gamma_{2} x_{2} a_{2}-\left(\lambda+\mu_{3}+\gamma_{1} x_{1}+\gamma_{2} x_{2}\right) a_{3}=-\gamma_{2} x_{2}$;

- система для нахождения значений $s_{k}$ :

$-\left(\lambda+\gamma_{1} x_{1}+\gamma_{2} x_{2}+\gamma_{3} x_{3}\right) s_{0}+\left(\lambda+\gamma_{1} x_{1}\right) s_{1}+\gamma_{2} x_{2} s_{2}+\gamma_{3} x_{3} s_{3}=-\gamma_{3} x_{3}$,

$-\left(\mu_{1}+\gamma_{2} x_{2}+\gamma_{3} x_{3}\right) s_{1}+\left(\mu_{1}+\gamma_{2} x_{2}\right) s_{2}+\gamma_{3} x_{3} s_{3}=-\gamma_{3} x_{3}$,

$\left(\lambda+\gamma_{1} x_{1}\right) s_{1}-\left(\lambda+\mu_{2}+\gamma_{1} x_{1}+\gamma_{3} x_{3}\right) s_{2}+\left(\mu_{2}+\gamma_{3} x_{3}\right) s_{3}=-\gamma_{3} x_{3}$,

$\mu_{3} s_{0}+\left(\lambda+\gamma_{1} x_{1}\right) s_{1}+\gamma_{2} x_{2} s_{2}-\left(\lambda+\mu_{3}+\gamma_{1} x_{1}+\gamma_{2} x_{2}\right) s_{3}=\lambda+\gamma_{1} x_{1}+\gamma_{2} x_{2}$.

Доказательство этой теоремы проводится аналогично доказательству теоремы 11 из [18].

Таким образом, асимптотическая характеристическая функция числа заявок на орбите в исследуемой RQ-системе является гауссовской:

$$
h\left(u_{1}, u_{2}, u_{3}\right)=\exp \left\{\sum_{\nu=1}^{3} j u_{\nu} x_{\nu}-\frac{1}{2} \sum_{\nu=1}^{3} \sum_{\kappa=1}^{3} u_{\kappa} u_{\nu} K_{\kappa \nu}\right\} .
$$

4. Численная реализация. Используя формулы, полученные выше для нахождения параметров гауссовского распределения, построим аппроксимацию распределения вероятности суммарного числа заявок на орбите.

Пусть $\sigma_{1}=\sigma_{2}=\sigma_{3}$. Из полученных параметров для трехмерного распределения вероятностей числа заявок на орбите перейдем к одномерному нормальному распределению с параметрами

$$
a=\frac{x_{1}+x_{2}+x_{3}}{\sigma}, \quad d=\frac{K_{11}+K_{22}+K_{33}+2 K_{12}+2 K_{13}+2 K_{23}}{\sigma} .
$$

Положим значения параметров системы следующими:

$$
\gamma_{1}=\gamma_{2}=\gamma_{3}=2, \quad \sigma=0.01, \quad \mu_{1}=2, \quad \mu_{2}=3, \quad \mu_{3}=4, \quad \lambda=0.5
$$

Отсюда

$$
R_{0}=0.458, \quad R_{1}=0.25, \quad R_{2}=0.167, \quad R_{3}=0.125
$$




$$
\begin{gathered}
x_{1}=0.136, \quad x_{2}=0.091, \quad x_{3}=0.068 \\
K_{11}=0.167, \quad K_{12}=0.035, \quad K_{13}=0.027 \\
K_{22}=0.1, \quad K_{23}=0.017, \quad K_{33}=0.077 .
\end{gathered}
$$

Тогда $a=29.5$ - математическое ожидание, $d=50.2$ - дисперсия.

На рис. 2 представлена аппроксимация распределения вероятностей $P(i)$ числа заявок на орбите, полученная из гауссовского распределения с параметрами $a, \sqrt{d}$.

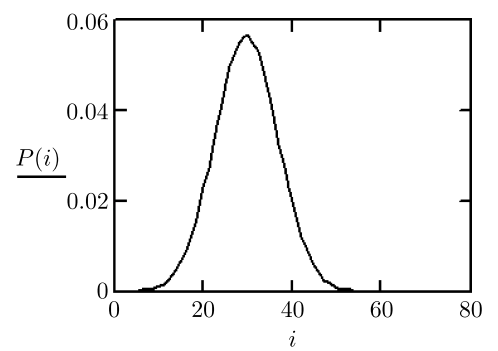

Рис. 2. Гауссовская аппроксимация распределения вероятностей $P(i)$ числа заявок на орбите

[Figure 2. Gaussian approximation of the probability distribution $P(i)$ of the number of customers in the orbit]

Заключение. В работе исследована RQ-система с вытеснением заявок и трехфазным пофазовым дообслуживанием. Для нее получено стационарное распределение вероятностей состояний прибора. Показано, что характеристическую функцию числа заявок на орбите можно аппроксимировать нормальным распределением. Найдены параметры этого распределения.

Вызывает интерес задача более общего плана, то есть система с повторами и дообслуживанием с произвольным распределением времени обслуживания.

Конкурирующие интересы. Заявляем, что в отношении авторства и публикации этой статьи конфликта интересов не имеем.

Авторская ответственность. Все авторы принимали участие в разработке концепции статьи и в написании рукописи. Авторы несут полную ответственность за предоставление окончательной рукописи в печать. Окончательная версия рукописи была одобрена всеми авторами.

Финансирование. Работа выполнена при финансовой поддержке РФФИ (проект № 18-01-00277 a).

Благодарность. Авторы благодарны рецензенту за тщательное прочтение статьи и ценные предложения и комментарии.

\section{Библиографический список}

1. Yang T., Templeton J.G.C. A survey on retrial queue// Queueing Syst., 1987. pp. 201-233. doi : 10.1007/BF01158899.

2. Falin G. I. A survey of retrial queues // Queueing Syst., 1990. vol. 7. pp. 127-168. doi: 10. 1007/BF01158472.

3. Falin G. I., Templeton J.G.C. Retrial Queues. London: Chapman and Hall, 1997. 338 pp.

4. Artalejo J. R., Choudhury G. Steady state snalysis of an M/G/1 queue with repeated attempts and two-phase service // Quality Technology and Quantitative Management, 2004. vol. 1, no. 2. pp. 189-199. doi: 10.1080/16843703.2004.11673072.

5. Choudhury G., Deka K. An M/G/1 retrial queueing system with two phases of service subject to the server breakdown and repair // Performance Evaluation, 2008. vol. 65, no. 10. pp. 714-724. doi: 10.1016/j.peva.2008.04.004.

6. Ke J. C., Choudhury G. A batch arrival retrial queue with general retrial times under Bernoulli vacation schedule for unreliable server and delaying repair // Appl. Math. Model., 2012. vol. 36, no. 1. pp. 255-269. doi: 10.1016/j.apm.2011.05.047. 
7. Kumar K., Vijayakumar A., Arivudainambi D. An M/G/1 retrial queueing system with two-phase service and preemptive resume// Ann. Oper. Res., 2002. vol.113. pp. 61-79. doi: 10.1023/A:1020901710087.

8. Kuki A., Wang J., Wang F., Sztrik J. Finite source retrial queues with two phase service // Int. J. Oper. Res., 2017. vol. 30, no.4. pp. 421-440. doi: 10.1504/IJOR. 2017.10008470.

9. Zhou Z. Analysis of M1, M2/M1, M2/N retrial queue system with non-preemptive priority / 2018 IEEE 9th International Conference on Software Engineering and Service Science (ICSESS). Beijing, China, 2018. pp. 301-304. doi: 10.1109/icsess.2018.8663929.

10. Korenevskaya M., Zayats O., Ilyashenko A., Muliukha V. Retrial queuing system with randomized push-out mechanism and non-preemptive priority // Procedia Computer Science, 2019. vol. 150. pp. 716-725. doi: 10.1016/j.procs.2019.02.016.

11. Senthil Kumar M., Chakravarthy S. R., Arumuganathan R. Preemptive resume priority retrial queue with two classes of MAP arrivals // Appl. Math. Sci., 2013. vol.7. pp. 25692589. doi: 10.12988/ams.2013.13231.

12. Lan S., Tang Y. Performance analysis of a discrete-time Geo/G/1 retrial queue with nonpreemptive priority, working vacations and vacation interruption // J. Ind. Manag. Optim., 2019. vol. 15, no. 3. pp. 1421-1446. doi: 10.3934/jimo. 2018102.

13. Devos A., Walraevens J., Bruneel H. A priority retrial queue with constant retrial policy / Y. Takahashi, T. Phung-Duc, S. Wittevrongel, W. Yue (eds.), Queueing Theory and Network Applications. QTNA 2018/ Lecture Notes in Computer Science, 10932. Cham: Springer, 2018. pp. 3-21. doi: 10.1007/978-3-319-93736-6_1.

14. Gao S. A preemptive priority retrial queue with two classes of customers and general retrial times // Oper. Res. Int. J., 2015. vol. 15. pp. 233-251. doi: 10.1007/s12351-015-0175-z.

15. Назаров А. А., Измайлова Я. Е. Исследование RQ-системы M|E2|1 с вытеснением заявок и сохранением фазовой реализации обслуживания // Вестн. Том. гос. унта. Управление, вычислительная техника и информатика, 2018. №42. С. 72-78. doi : 10.17223/19988605/42/8.

16. Назаров А. А., Измайлова Я. Е. Исследование RQ-системы $M^{(2)}\left|B(x)^{(2)}\right| 1$ с $R$-настойчивым вытеснением альтернативных заявок // Вестник СибГАУ, 2016. Т. 17, № 2 . C. 328-334.

17. Назаров А. А., Черникова Я. Е. Исследование RQ-системы M|GI|1 с вытеснением в условии большой задержки // Известия Томского политехнического университета, 2013. Т. 323, № 5. С. 16-20.

18. Измайлова Я. Е. Исследование математических моделей $R Q$-систем с вытеснением залвок: Дис. ... канд. физ.-мат. наук. Томск, 2017. 148 с. 
MSC: $60 \mathrm{~K} 20,41 \mathrm{~A} 60$

\title{
Research of a retrial queueing system with exclusion of customers and three-phase phased by follow-up
}

\author{
A. A. Nazarov, Ya. E. Izmailova \\ National Research Tomsk State University, \\ Institute of Applied Mathematics and Computer Sciences, \\ 36, Lenin ave., Tomsk, 634050, Russian Federation.
}

\begin{abstract}
In this paper, we consider a retrial queueing system (RQ-system) which receives to the input a Poisson flow with a given intensity. If at the time of customer the server is busy, the displacement of customer standing on the server takes place. Customers that do not have time to be successfully serviced go into orbit, in order to, after an accidental exponential delay, again turn to the server for maintenance. It is shown that the limiting characteristic function of the number of customers in the orbit and the states of the server converges to a three-dimensional Gaussian distribution. The mean vector and covariance matrix are obtained for this distribution. A stationary probability distribution of the server states is also found.
\end{abstract}

Keywords: retrial queueing system, exclusion of customers, three-phase service, follow-up customers, Gaussian approximation, asymptotic analysis.

Received: $18^{\text {th }}$ November, 2019 / Revised: $26^{\text {th }}$ May, $2020 /$

Accepted: $1^{\text {st }}$ June, $2020 /$ First online: $3^{\text {rd }}$ June, 2020

Competing interests. We declare that we have no conflicts of interest in the authorship and publication of this article.

Author's Responsibilities. Each author has participated in the article concept development and in the manuscript writing. The authors are absolutely responsible for submitting the final manuscript in print. Each author has approved the final version of manuscript.

Funding. This work was supported by the Russian Foundation for Basic Research (projects no. 18-01-00277 a).

\section{Research Article}

()(7) The content is published under the terms of the Creative Commons Attribution 4.0 International License (http://creativecommons.org/licenses/by/4.0/)

Please cite this article in press as:

Nazarov A. A., Izmailova Ya. E. Research of a retrial queueing system with exclusion of customers and three-phase phased by follow-up, Vestn. Samar. Gos. Tekhn. Univ., Ser. Fiz.Mat. Nauki [J. Samara State Tech. Univ., Ser. Phys. Math. Sci.], 2020, vol. 24, no. 2, pp. 331-342. doi: 10.14498/vsgtu1756 (In Russian).

\section{Authors' Details:}

Anatolii A. Nazarov (1D https://orcid.org/0000-0002-5097-5629

Dr. Tech. Sci., Professor; Head of Department; Dept. of Probability Theory and Mathematical Statistic; e-mail: nazarov.tsu@gmail.com

Yana E. Izmailova (10) https://orcid.org/0000-0002-9132-0127

Cand. Phys. \& Math. Sci.; Associate Professor; Dept. of Probability Theory and Mathematical Statistic; e-mail: evgenevna.92@mail.ru 
Acknowledgments. The authors are grateful to the reviewer for careful reading of the paper and valuable suggestions and comments.

\section{References}

1. Yang T., Templeton J.G.C. A survey on retrial queue, Queueing Syst., 1987, pp. 201-233. doi : 10.1007/BF01158899.

2. Falin G. I. A survey of retrial queues, Queueing Syst., 1990, vol. 7, pp. 127-168. doi: 10. 1007/BF01158472.

3. Falin G. I., Templeton J.G.C. Retrial Queues. London, Chapman and Hall, 1997, 338 pp.

4. Artalejo J. R., Choudhury G. Steady state snalysis of an M/G/1 queue with repeated attempts and two-phase service, Quality Technology and Quantitative Management, 2004, vol. 1, no. 2, pp. 189-199. doi : 10.1080/16843703.2004.11673072.

5. Choudhury G., Deka K. An M/G/1 retrial queueing system with two phases of service subject to the server breakdown and repair, Performance Evaluation, 2008, vol. 65, no. 10, pp. 714-724. doi: 10.1016/j . peva.2008.04.004.

6. Ke J. C., Choudhury G. A batch arrival retrial queue with general retrial times under Bernoulli vacation schedule for unreliable server and delaying repair, Appl. Math. Model., 2012, vol. 36, no. 1, pp. 255-269. doi: 10.1016/j.apm.2011.05.047.

7. Kumar K., Vijayakumar A., Arivudainambi D. An M/G/1 retrial queueing system with twophase service and preemptive resume, Ann. Oper. Res., 2002, vol.113, pp. 61-79. doi: 10. 1023/A : 1020901710087.

8. Kuki A., Wang J., Wang F., Sztrik J. Finite source retrial queues with two phase service, Int. J. Oper. Res., 2017, vol.30, no. 4, pp. 421-440. doi : 10.1504/IJOR.2017.10008470.

9. Zhou Z. Analysis of M1, M2/M1, M2/N retrial queue system with non-preemptive priority, In: 2018 IEEE 9th International Conference on Software Engineering and Service Science (ICSESS). Beijing, China, 2018, pp. 301-304. doi: 10.1109/icsess.2018.8663929.

10. Korenevskaya M., Zayats O., Ilyashenko A., Muliukha V. Retrial queuing system with randomized push-out mechanism and non-preemptive priority, Procedia Computer Science, 2019, vol. 150, pp. 716-725. doi: 10.1016/j.procs.2019.02.016.

11. Senthil Kumar M., Chakravarthy S. R., Arumuganathan R. Preemptive resume priority retrial queue with two classes of MAP arrivals, Appl. Math. Sci., 2013, vol.7, pp. 25692589. doi: 10.12988/ams. 2013.13231.

12. Lan S., Tang Y. Performance analysis of a discrete-time Geo/G/1 retrial queue with nonpreemptive priority, working vacations and vacation interruption, J. Ind. Manag. Optim., 2019, vol. 15, no. 3, pp. 1421-1446. doi: 10.3934/jimo. 2018102.

13. Devos A., Walraevens J., Bruneel H. A priority retrial queue with constant retrial policy, In: Y. Takahashi, T. Phung-Duc, S. Wittevrongel, W. Yue (eds.), Queueing Theory and Network Applications. QTNA 2018, Lecture Notes in Computer Science, 10932. Cham, Springer, 2018, pp. 3-21. doi: 10.1007/978-3-319-93736-6_1.

14. Gao S. A preemptive priority retrial queue with two classes of customers and general retrial times, Oper. Res. Int. J., 2015, vol. 15, pp. 233-251. doi : 10.1007/s12351-015-0175-z.

15. Nazarov A. A., Izmailova Ya. E. Research of RQ-system M|E2|1 with request displacement and conserving phase realization of servicin, Tomsk State University Journal of Control and Computer Science, 2018, no. 42, pp. 72-78 (In Russian). doi: 10.17223/19988605/42/8.

16. Nazarov A. A., Izmailova Ya. E. Research of the retrial queueing system $M^{(2)}\left|B(x)^{(2)}\right| 1$ with $R$-persistent exclusion of alternative customers, Vestnik of the Siberian State Aerospace University, 2016, vol. 17, no. 2, pp. 328-334 (In Russian).

17. Nazarov A. A., Chernikova Ya. E. Research of retrial queueing system M $\mid$ GI $\mid 1$ with the exclusion customers in the condition of long delays, Bulletin of the Tomsk Polytechnic University, 2013, vol. 323, no. 5, pp. 16-20 (In Russian).

18. Izmailova Ya. E. Research of mathematical models of RQ-systems with displacement of customers, Cand. Phys.-Math. Sci. Diss. Tomsk, 2017, 148 pp. (In Russian) 